\title{
ON THE NUMBER OF EULER TRAILS IN DIRECTED GRAPHS
}

\author{
JAKOB JONSSON
}

\begin{abstract}
Let $G$ be an Eulerian digraph with all in- and out-degrees equal to 2, and let $\pi$ be an Euler trail in $G$. We consider an intersection matrix $\mathbf{L}(\pi)$ with the property that the determinant of $\mathbf{L}(\pi)+\mathbf{I}$ is equal to the number of Euler trails in $G$; $\mathbf{I}$ denotes the identity matrix. We show that if the inverse of $\mathbf{L}(\pi)$ exists, then $\mathbf{L}^{-1}(\pi)=\mathbf{L}(\sigma)$ for a certain Euler trail $\sigma$ in $G$. Furthermore, we use properties of the intersection matrix to prove some results about how to divide the set of Euler trails in a digraph into smaller sets of the same size.
\end{abstract}

\section{Introduction}

The aim of this article is to study enumerative properties of Euler trails in digraphs (or, more precisely, directed multigraphs with loops permitted). A digraph $G=(V, A, t, h)$ consists of a set $V=V_{G}$ of vertices, a set $A=A_{G}$ of $\operatorname{arcs}$, and two functions $t, h: A_{G} \rightarrow V_{G} ; t(a)=t_{G}(a)$ is the tail of the arc $a$ and $h(a)=h_{G}(a)$ is the head of $a$. Intuitively speaking, an arc is an arrow pointing from its tail to its head.

Let $S_{A}$ be the symmetric group of permutations of $A$ with multiplication defined as $\pi \sigma(a)=\pi(\sigma(a))$. A permutation $\pi \in S_{A}$ is a $G$-permutation if $h(a)=t(\pi(a))$ for all $a \in A$; this means that $a$ ends where $\pi(a)$ begins. The permutation $\pi$ is an Euler trail in $G$ if $\pi$ is a cyclic $G$-permutation. A digraph containing Euler trails is an Eulerian digraph. For $m>0$, an $m$ regular Eulerian digraph is an Eulerian digraph where, for each vertex $v$, there are exactly $m$ arcs with head $v$ (and hence $m$ arcs with tail $v$ ). Some 2-regular Eulerian digraphs are illustrated in Figures 6 and 7 in Section 5.

In Section 3, we consider a 2-regular Eulerian digraph $G$ with vertex set $[n]:=\{1, \ldots, n\}$ and with arc set $\{+1, \ldots,+n,-1, \ldots,-n\}$, where the head of $\pm k$ is $k$. Let $\pi$ be an Euler trail in $G$. Say that the vertices $j$ and $k$ intersect if $\pi \circ(+j,-j)(+k,-k)$ is an Euler trail and $j \neq k$. Cohn and Lempel [4] defined an $n \times n$ intersection matrix

$$
\mathbf{L}(\pi)=\left(l_{j k}\right)_{j, k \in[n]}
$$

Received October 28, 1996; in revised form April 26, 1999. 
of $\pi$ by letting $l_{j k}$ be 1 if $j$ and $k$ intersect in $\pi$ and 0 otherwise. They showed for any $J \subseteq[n]$ that the nullity of

$$
\mathbf{L}_{J}(\pi)=\left(l_{j k}\right)_{j, k \in J}
$$

over $G F(2)$ is equal to the number of cycles minus one in the $G$-permutation $\sigma=\pi \circ \prod_{j \in J}(+j,-j)$. Beck [2] generalized this result for not necessarily disjoint transpositions.

Macris \& Pulé [11] and [12] demonstrated how to give the elements in $\mathbf{L}(\pi)$ signs so that $\operatorname{det} \mathbf{L}_{J}(\pi) \in\{0,1\}$ over Z; $\operatorname{det} \mathbf{L}_{J}(\pi)=1$ if and only if $\pi \circ \prod_{j \in J}(+j,-j)$ is an Euler trail. Actually, they proved that $\operatorname{det}(\mathbf{L}(\pi)+\mathbf{I})$ is equal to the number of Euler trails in $G$, where $\mathbf{I}$ is the identity matrix (Theorem 3.1). Lauri [10] carried out the details (Theorem 3.2) using methods similar to those used by Cohn \& Lempel in [4].

One of our own contributions in Section 3 is the following result (Theorem 3.4): If $\sigma=\pi \circ \prod_{j \in J}(+j,-j)$ is an Euler trail, then $\mathbf{L}_{J}(\sigma)=\mathbf{L}_{J}^{-1}(\pi)$. In [8], we generalize the intersection matrix to arbitrary Eulerian digraphs.

In Section 4, we consider a (not necessarily 2-regular) Eulerian digraph $G$ in which the vertices can be divided into sets $U_{1}, \ldots, U_{\varphi}$ and into sets $W_{1}, \ldots, W_{\varphi}$ such that if $a$ is an arc with its tail in $U_{k}$, then the head of $a$ is in $W_{k}$. Another way of expressing this is that $t^{-1}\left(U_{k}\right)=h^{-1}\left(W_{k}\right)$ for every $k$.

Let $\pi$ be a fixed Euler trail in $G$, and let $\sigma$ be another Euler trail in $G$. Then $\sigma^{-1} \pi$ is a permutation of the arcs such that the set $A_{k}$ of arcs with heads in $W_{k}$ is mapped onto itself for all $k$. In particular, $\sigma^{-1} \pi$ can be restricted to $A_{k}$. Let $s_{k}$ be the sign of this restricted permutation. We obtain a sequence $\left(s_{1}, \ldots, s_{\varphi-1}\right)$ of signs associated to the Euler trail $\sigma$; we omit the $\varphi$ th sign, since it is uniquely determined by (and equal to) $\prod_{i=1}^{\varphi-1} s_{k}$.

We prove that the number of Euler trails with a given sequence of signs is independent of the sequence, that is, the number is the same for all $2^{\varphi-1}$ possible sequences (Theorem 4.2). Suppose in addition that $G$ is 2-regular, and let $v$ and $w$ be vertices such that there is an arc from $v$ to $w$. Then the number of Euler trails with a given sequence of signs and with $v$ and $w$ intersecting is again constant (Theorem 4.3).

In Section 5, the article is concluded with a discussion about arc digraphs. In an arc digraph, there are sets $U_{1}, \ldots, U_{\varphi}, W_{1}, \ldots, W_{\varphi}$ with the property that for each $k \in[\varphi], u \in U_{k}$, and $w \in W_{k}$, there is exactly one arc with tail $u$ and head $w$. The most famous Eulerian arc digraphs are perhaps the 2-regular de Bruijn digraphs, in which Euler trails can be identified with binary de Bruijn sequences. For details, consider Subsection 5.2, where we apply Theorem 4.2 to de Bruijn sequences. 
The author thanks Svante Linusson and an anonymous referee for valuable comments and suggestions.

\section{Some group theory}

To obtain decent formulations and proofs of our results, some group theory will be useful. Namely, there is an obvious group-theoretic interpretation of $G$-permutations as follows.

Let $G$ be an Eulerian digraph. For each vertex $v \in V$, note that $h^{-1}(v)$ is the set of arcs with head $v$. Let $T_{G}$ be the subgroup of $S_{A}$ consisting of all permutations $\sigma$ such that $\sigma\left(h^{-1}(v)\right)=h^{-1}(v)$ for all $v \in V$. Clearly $T_{G}$ is a group of the form $\prod_{v \in V} S_{h^{-1}(v)}$. Let $\pi$ be a fixed $G$-permutation; we claim that $\pi T_{G}$ is the set of $G$-permutations. Namely, for any $\tau \in T_{G}, \pi \tau$ is also a $G$-permutation, since $t(\pi \tau(a))=h(\tau(a))=h(a)$. Moreover, if $\sigma$ is a $G$-permutation, then $\pi^{-1} \sigma \in T_{G}$ since $h\left(\pi^{-1} \sigma(a)\right)=t(\sigma(a))=h(a)$.

Conversely, every left coset $\pi T$ of a permutation group of the form $T=$ $\prod_{v \in V} S_{A(v)}$, where the sets $A(v)$ are disjoint, can be interpreted as the set of $G$ permutations in a certain digraph $G$. Namely, put $V_{G}=V, A_{G}=\bigcup_{v \in V} A(v)$, $h(a)=v$ for all $a \in A(v)$, and $t(a)=h\left(\sigma^{-1}(a)\right)$ for arbitrarily chosen $\sigma \in \pi T$. This is well defined since, with $\sigma=\pi \tau$, we obtain that

$$
h\left(\sigma^{-1}(a)\right)=h\left(\tau^{-1} \pi^{-1}(a)\right)=h\left(\pi^{-1}(a)\right) .
$$

We say that $G$ is the digraph induced by $\pi T$.

For a permutation $\pi \in S_{A}$, let $c(\pi)$ denote the number of cycles minus one in $\pi$; hence $\pi$ is a cyclic permutation if and only if $c(\pi)=0$. Frequently, we will consider restrictions of a permutation to smaller sets. In those cases we will find it necessary to write $c_{A}(\pi)$ instead of $c(\pi)$. Namely, if $B$ is a subset of $A$ such that $\pi(B)=B$, then both $c_{A}(\pi)$ and $c_{B}(\pi)$ are defined since $\pi$ can be restricted to a permutation in $S_{B}$.

Let $B \subset A$, where $A$ is a finite set. We may consider $S_{B}$ as a subgroup of $S_{A}$ by defining $\pi(a)=a$ for all $\pi \in S_{B}$ and $a \in A \backslash B$. The following construction will be used frequently throughout this article. For $\pi \in S_{A}$, let $\pi^{B} \in S_{B}$ be the permutation obtained from $\pi$ by "removing" all elements not in $B$ from the cycle decomposition of $\pi$. More precisely, for each $b \in B$, set

$$
\pi^{B}(b)=\pi^{k}(b),
$$

where $k$ is the smallest positive number $j$ such that $\pi^{j}(b) \in B$. If $\pi(B)=B$, then $\pi^{B}$ is the restriction of $\pi$ to the set $B$. 
ExAmPle 2.1. Let $A=\{1, \ldots, 18\}$,

$$
\begin{aligned}
& \pi=(1,3,9,8,5,15,7,2,6,18,16,10,11,14,4,12,17,13), \\
& \sigma=(1,8,7,18,11,12)(2,16,14,17,3,5)(4,13,15,6)(9,10),
\end{aligned}
$$

and $B=\{1,7,8,9,10,11,12,18\}$. Then

$$
\pi^{B}=(1,9,8,7,18,10,11,12)
$$

and

$$
\sigma^{B}=(1,8,7,18,11,12)(9,10) .
$$

Note that $\sigma^{B}$ is the restriction of $\sigma$ to $B$.

$$
\text { If } \pi, \sigma \in S_{A} \text { and } \pi(a)=\sigma(a) \text { for all } a \notin B \text {, then }
$$

$$
\left(\sigma^{B}\right)^{-1} \pi^{B}=\sigma^{-1} \pi,
$$

where the right-hand expression is restricted to $B$. To prove this, first assume that $B=A \backslash\{a\}$. Then $\pi(b)=\pi^{B}(b)$ unless $\pi(b)=a$, in which case $\pi^{B}(b)=\pi(a)=\sigma(a)$. The same is true for $\sigma$; hence

$$
\left(\sigma^{B}\right)^{-1} \pi^{B}(b)=\left(\sigma^{B}\right)^{-1}(\sigma(a))=\sigma^{-1}(a)=\sigma^{-1} \pi(b) .
$$

To prove (2) for general $B$, use induction over $|A \backslash B|(|J|$ is the number of elements in the set $J$ ).

For more information about permutation groups, see [5].

\section{The intersection matrix}

The aim of this section is to describe the intersection matrix of an Euler trail in a 2-regular digraph. Some small modifications compared to [10]-[12] are made to make it possible to compare the intersection matrices for different Euler trails.

\subsection{Definition and known results}

Let $n>0$ be an integer; let $G$ be a 2-regular Eulerian digraph with vertex set $V_{G}=[n]$ and arc set $A_{G}=\{+1, \ldots,+n,-1, \ldots,-n\}$, where the head of the $\operatorname{arcs}+k$ and $-k$ is $k$. Let $\pi$ be an Euler trail in $G$. Define the intersection matrix $\mathbf{L}(\pi)=\left\{l_{j k}: j, k \in[n]\right\}$ of $\pi$ as follows. For $j, k \in[n]$, put $B=$ $\{+j,-j,+k,-k\}$ and

$$
l_{j k}=\left\{\begin{aligned}
1 & \text { if } \pi^{B}=(-j,+k,+j,-k) \\
-1 & \text { if } \pi^{B}=(-j,-k,+j,+k) \\
0 & \text { otherwise. }
\end{aligned}\right.
$$


In particular, $l_{j j}=0$. Note that $l_{j k}$ is nonzero if and only if $j$ and $k$ intersect in the sense described in the introduction.

If instead $V_{G}=\left\{j_{1}, \ldots, j_{k}\right\} \subset[n]$, then we may define the $k \times k$ matrix $\mathbf{L}(\pi)$ in exactly the same manner with rows and columns corresponding to $j_{1}, \ldots, j_{k}$ instead of $1, \ldots, n$.

One easily checks that our definition of $\mathbf{L}(\pi)$ is the same as the corresponding definitions in [12] and [10]. See Subsection 3.3 for another equivalent definition.

First, we state a few known results about the intersection matrix.

TheOREM 3.1 (Macris \& Pulé [12]). Let $\pi$ be an Euler trail in the 2-regular digraph $G$. Then the number of Euler trails in $G$ is equal to

$$
\operatorname{det}(\mathbf{L}(\pi)+\mathbf{I}),
$$

where $\mathbf{I}$ is the identity matrix.

For any set $J \subseteq[n]$, put $\tau_{J}=\prod_{j \in J}(+j,-j)$; this notation will be used throughout Section 3. Since $T_{G}$ is the abelian group generated by the transpositions $(+1,-1), \ldots,(+n,-n)$, it is clear that $\pi \tau_{J}$ is a $G$-permutation whenever $\pi$ is a $G$-permutation. Moreover, $c\left(\pi \tau_{J}\right)$ is even if and only if $|J|$ is even. In particular, if $\pi \tau_{J}$ is an Euler trail, then $|J|$ is even. Put

$$
\mathbf{L}_{J}(\pi)=\left(l_{i j}\right)_{i, j \in J} .
$$

TheOREM 3.2 (Lauri [10]). Let $\pi$ be an Euler trail in a 2-regular digraph with vertex set $[n]$. Then for any set $J \subseteq[n]$,

$$
\operatorname{det} \mathbf{L}_{J}(\pi)= \begin{cases}1 & \text { if } \pi \tau_{J} \text { is an Euler trail } \\ 0 & \text { otherwise. }\end{cases}
$$

We conclude this summary of known results by mentioning a result about the rank of the matrix $\mathbf{L}_{J}(\pi)$. To prove it, follow Cohn \& Lempel [4] with appropriate replacements of $\mathrm{GF}(2)$-matrices with Z-matrices.

THEOREM 3.3 (Cohn \& Lempel [4]). Let $\pi$ be an Euler trail in a 2-regular digraph with vertex set $[n]$. Then for any $J \subseteq[n]$,

$$
c_{A}\left(\pi \tau_{J}\right)=|J|-\operatorname{rank} \mathbf{L}_{J}(\pi)=\text { the nullity of } \mathbf{L}_{J}(\pi) .
$$




\subsection{On the inverse of the intersection matrix}

The results of this subsection will not be used in later sections. We will prove a result that requires our definition of the intersection matrix. Namely, to construct the intersection matrix, one must give the arcs signs. In this paper these signs are fixed from the beginning, while in [10] the signs are not necessarily the same in different Euler trails.

TheORem 3.4. Let $\pi$ be an Euler trail in a 2-regular digraph with vertex set $[n]$, and let $J \subseteq[n]$ be such that $\pi \tau_{J}$ is an Euler trail. Then

$$
\mathbf{L}_{J}\left(\pi \tau_{J}\right)=\mathbf{L}_{J}^{-1}(\pi) .
$$

We will in fact prove more than Theorem 3.4:

Lemma 3.5. With notations and assumptions as in Theorem 3.4, suppose that $J=[2 m](2 m \leq n)$. Write

$$
\mathbf{L}(\pi)=\left(\begin{array}{cc}
\mathbf{L}_{J} & -\mathbf{A}^{\mathrm{T}} \\
\mathbf{A} & \mathbf{B}
\end{array}\right),
$$

where $\mathbf{L}_{J}=\mathbf{L}_{J}(\pi)$, and put

$$
\mathbf{M}(\pi, J)=\left(\begin{array}{cc}
\mathbf{L}_{J} & \mathbf{0} \\
\mathbf{A} & \mathbf{I}
\end{array}\right) .
$$

Then

$$
\mathbf{L}(\pi)+\mathbf{I}=\mathbf{M}(\pi, J) \cdot\left(\mathbf{L}\left(\pi \tau_{J}\right)+\mathbf{I}\right)
$$

or, equivalently,

$$
\mathbf{L}\left(\pi \tau_{J}\right)=\left(\begin{array}{cc}
\mathbf{L}_{J}^{-1} & -\mathbf{L}_{J}^{-1} \mathbf{A}^{\mathrm{T}} \\
-\mathbf{A} \mathbf{L}_{J}^{-1} & \mathbf{B}+\mathbf{A} \mathbf{L}_{J}^{-1} \mathbf{A}^{\mathrm{T}}
\end{array}\right) .
$$

Proof. First consider $J=\{1,2\}$. We have to show that (4) holds. However, note that $l_{i j}\left(\pi \tau_{J}\right)$ only depends on $1,2, i$, and $j$. Thus it suffices to consider $n=4$; in particular, there is only a finite number of cases, and these are easily checked. As in [10], we let the reader do this. Using the row vectors $\mathbf{R}_{i}$ introduced later in Subsection 3.3, one may carry out a rigorous (but cumbersome) proof.

Induction over $m$ is used to prove (3); suppose that $m>1$. There exist distinct numbers $i, j \leq 2 m$ such that $\pi_{1}=\pi \tau_{J \backslash\{i, j\}}$ is a cyclic permutation. 
Namely, otherwise $\mathbf{L}_{J}\left(\pi \tau_{J}\right)$ would be the zero matrix. Assuming (without loss of generality) that $i=1$ and $j=2$, we may write

$$
\mathbf{L}\left(\pi_{1}\right)=\left(\begin{array}{ccc}
\mathbf{D} & -\mathbf{P}^{\mathrm{T}} & -\mathbf{Q}^{\mathrm{T}} \\
\mathbf{P} & \mathbf{E} & -\mathbf{R}^{\mathrm{T}} \\
\mathbf{Q} & \mathbf{R} & \mathbf{S}
\end{array}\right),
$$

where $\mathbf{D}=\mathbf{L}_{\{1,2\}}\left(\pi_{1}\right), \mathbf{E}=\mathbf{L}_{\{3, \ldots, 2 m\}}\left(\pi_{1}\right)$; the other matrices are defined in the obvious manner.

By induction,

$$
\mathbf{L}\left(\pi_{1}\right)+\mathbf{I}=\mathbf{M}\left(\pi_{1},\{1,2\}\right) \cdot\left(\mathbf{L}\left(\pi \tau_{J}\right)+\mathbf{I}\right)
$$

and

$$
\mathbf{L}\left(\pi_{1}\right)+\mathbf{I}=\mathbf{M}\left(\pi_{1}, J \backslash\{1,2\}\right) \cdot(\mathbf{L}(\pi)+\mathbf{I}),
$$

where

$$
\mathbf{M}\left(\pi_{1}, J \backslash\{1,2\}\right)=\left(\begin{array}{ccc}
\mathbf{I} & -\mathbf{P}^{\mathrm{T}} & \mathbf{0} \\
\mathbf{0} & \mathbf{E} & \mathbf{0} \\
\mathbf{0} & \mathbf{R} & \mathbf{I}
\end{array}\right)
$$

This implies that

$$
\mathbf{L}(\pi)+\mathbf{I}=\mathbf{M}^{-1}\left(\pi_{1}, J \backslash\{1,2\}\right) \cdot \mathbf{M}\left(\pi_{1},\{1,2\}\right) \cdot\left(\mathbf{L}\left(\pi \tau_{J}\right)+\mathbf{I}\right) .
$$

Some easy computations yield that $\mathbf{M}^{-1}\left(\pi_{1}, J \backslash\{1,2\}\right) \cdot \mathbf{M}\left(\pi_{1},\{1,2\}\right)$ is equal to

$$
\left(\begin{array}{ccc}
\mathbf{D}+\mathbf{P}^{\mathrm{T}} \mathbf{E}^{-1} \mathbf{P} & \mathbf{P}^{\mathrm{T}} \mathbf{E}^{-1} & \mathbf{0} \\
\mathbf{E}^{-1} \mathbf{P} & \mathbf{E}^{-1} & \mathbf{0} \\
\mathbf{Q}-\mathbf{R} \mathbf{E}^{-1} \mathbf{P} & -\mathbf{R} \mathbf{E}^{-1} & \mathbf{I}
\end{array}\right)
$$

Computing $\mathbf{L}(\pi)=\mathbf{M}^{-1}\left(\pi_{1}, J \backslash\{1,2\}\right) \cdot\left(\mathbf{L}\left(\pi_{1}\right)+\mathbf{I}\right)-\mathbf{I}$, we immediately realize that (5) is equal to $\mathbf{M}(\pi, J)$, which is exactly what we need to prove (3) and (4).

REMARK 3.6. One may note that the lower right block in (4) does not have the same shape in [10]. This depends on the fact that Lauri defines the matrix corresponding to $\pi \tau_{J}$ in a slightly different way. However, the fact that Lauri considers $\tau_{J} \pi$ instead of $\pi \tau_{J}$ only affects the signs in the lower left and upper right blocks. By the way, (4) is the resulting matrix after the first step in a two-step method of computing the inverse of $\mathbf{L}(\pi)$ (if the inverse exists); see for example [6], pp. 161-163. 
Remark 3.7. In [8], we investigate the matrices $\mathbf{L}(\pi)$ and $\mathbf{M}(\pi, J)$ further. We will show that the rows in $\mathbf{L}(\pi)$ can be interpreted as the vectors in a basis for a certain "cycle space". The matrix $\mathbf{M}(\pi, J)$ is the transformation matrix between two sets of bases corresponding to the Euler trails $\pi$ and $\pi \tau_{J}$.

\subsection{An application of the intersection matrix}

We proceed by giving an alternative way of defining the intersection matrix of an Euler trail. Using this new definition, we prove a result that will be used and generalized in Section 4.

As usual we consider a 2-regular digraph $G$ with vertex set $[n]$ and with $h^{-1}(i)=\{+i,-i\}$ for $i \in[n]$. Let $\pi$ be an Euler trail in $G$. Put

$$
\mathbf{e}_{i}=(0, \ldots, 0,1,0, \ldots, 0) \in \mathbf{Z}^{n}
$$

the unit element in $\mathbf{e}_{i}$ is on position $i(1 \leq i \leq n)$. Choose an arbitrary arc $a \in A$. Define the row vectors $\mathbf{R}_{b}(a, \pi)$ and $\mathbf{L}_{j}(\pi)(b \in A, j \in[n])$ as follows.

$$
\begin{aligned}
\mathbf{R}_{a}(a, \pi) & =(0, \ldots, 0), \\
\mathbf{R}_{\pi^{i+1}(a)}(a, \pi) & =\mathbf{R}_{\pi^{i}(a)}(a, \pi)+\operatorname{sgn}\left(\pi^{i}(a)\right) \cdot \mathbf{e}_{\left|\pi^{i}(a)\right|},
\end{aligned}
$$

$(0<i<2 n)$ and

$$
\mathbf{L}_{j}(\pi)=\mathbf{R}_{+j}(a, \pi)-\mathbf{R}_{-j}(a, \pi)+\mathbf{e}_{j} .
$$

The validity of the following statement is easily checked.

Proposition 3.8.

$$
\mathbf{L}(\pi)=\left(\begin{array}{c}
\mathbf{L}_{1}(\pi) \\
\mathbf{L}_{2}(\pi) \\
\mathbf{L}_{3}(\pi) \\
\vdots \\
\mathbf{L}_{n}(\pi)
\end{array}\right)
$$

is the intersection matrix of $\pi$.

Example 3.9. Consider the Euler trail

$$
\pi=(-1,-2,-4,+2,-5,+4,-3,+1,+3,+5) .
$$


We obtain the following table.

$$
\begin{array}{lll}
i & \pi^{i}(-1) & \mathbf{R}_{\pi^{i}(-1)} \\
0 & -1 & (0,0,0,0,0) \\
1 & -2 & (-1,0,0,0,0) \\
2 & -4 & (-1,-1,0,0,0) \\
3 & +2 & (-1,-1,0,-1,0) \\
4 & -5 & (-1,0,0,-1,0) \\
5 & +4 & (-1,0,0,-1,-1) \\
6 & -3 & (-1,0,0,0,-1) \\
7 & +1 & (-1,0,-1,0,-1) \\
8 & +3 & (0,0,-1,0,-1) \\
9 & +5 & (0,0,0,0,-1)
\end{array}
$$

Thus

$$
\mathbf{L}(\pi)=\left(\begin{array}{rrrrr}
0 & 0 & -1 & 0 & -1 \\
0 & 0 & 0 & -1 & 0 \\
1 & 0 & 0 & 0 & 0 \\
0 & 1 & 0 & 0 & -1 \\
1 & 0 & 0 & 1 & 0
\end{array}\right)
$$

THEOREM 3.10. Let $G$ be a 2-regular digraph and suppose that there are two vertex sets $U=\left\{u_{1}, \ldots, u_{k}\right\}$ and $W=\left\{w_{1}, \ldots, w_{k}\right\}(k>0)$ such that there is an arc with tail $u_{i}$ and head $w_{i}$ as well as an arc with tail $u_{i}$ and head $w_{i-1}$ for every $i \in[k] ; w_{0}=w_{k}$. Let $\pi$ be an Euler trail in G. Put $\mathbf{L}^{+}=\mathbf{L}(\pi)+\mathbf{I}, \mathbf{L}^{-}=\mathbf{L}(\pi)-\mathbf{I}$. Then there exist numbers $\mu_{i}, v_{i} \in\{-1,+1\}$, $i=1, \ldots, k$, such that

$$
\sum_{i=1}^{k} \mu_{i} \mathbf{L}_{u_{i}}^{+}=\sum_{i=1}^{k} v_{i} \mathbf{L}_{w_{i}}^{-}
$$

Moreover, if $U$ and $W$ are disjoint, then the number of Euler trails $\sigma$ in $G$ such that $\left(\sigma^{-1} \pi\right)^{h^{-1}(W)}$ is odd is equal to the number of Euler trails $\sigma$ such that $\left(\sigma^{-1} \pi\right)^{h^{-1}(W)}$ is even.

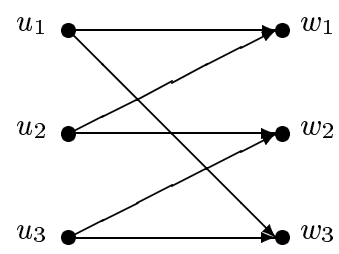

FIgURE 1. The case $k=3$ 
Proof. With customary choices of numbers $\mu_{i}, v_{i} \in\{-1,1\}$,

$$
\pi\left(\mu_{i} u_{i}\right)=v_{i} w_{i}, \pi\left(-\mu_{i} u_{i}\right)=-v_{i-1} w_{i-1}
$$

for all $i \in[k] ; v_{0}=v_{k}$ and $w_{0}=w_{k}$. This implies that

$$
\begin{aligned}
-\left(\mathbf{R}_{v_{i} w_{i}}-\mathbf{R}_{\mu_{i} u_{i}}\right) & =-\mu_{i} \mathbf{e}_{u_{i}} ; \\
\mathbf{R}_{-v_{i-1} w_{i-1}}-\mathbf{R}_{-\mu_{i} u_{i}} & =-\mu_{i} \mathbf{e}_{u_{i}} .
\end{aligned}
$$

Summing the left-hand sides, we obtain

$$
\begin{aligned}
\sum_{i=1}^{k}( & \left.\left(\mathbf{R}_{v_{i} w_{i}}-\mathbf{R}_{\mu_{i} u_{i}}\right)-\mathbf{R}_{-\mu_{i} u_{i}}+\mathbf{R}_{-v_{i-1} w_{i-1}}\right) \\
= & \sum_{i=1}^{k}\left(-\left(\mathbf{R}_{v_{i} w_{i}}-\mathbf{R}_{\mu_{i} u_{i}}\right)+\mathbf{R}_{-v_{i} w_{i}}-\mathbf{R}_{-\mu_{i} u_{i}}\right) \\
= & \sum_{i=1}^{k}\left(\mathbf{R}_{\mu_{i} u_{i}}-\mathbf{R}_{-\mu_{i} u_{i}}\right)-\sum_{i=1}^{k}\left(\mathbf{R}_{v_{i} w_{i}}-\mathbf{R}_{-v_{i} w_{i}}\right) \\
= & \sum_{i=1}^{k} \mu_{i} \mathbf{L}_{u_{i}}^{+}-2 \sum_{i=1}^{k} \mu_{i} \mathbf{e}_{u_{i}}-\sum_{i=1}^{k} v_{i} \mathbf{L}_{w_{i}}^{-} .
\end{aligned}
$$

Summing the right-hand sides, we obtain $-2 \sum_{i=1}^{k} \mu_{i} \mathbf{e}_{u_{i}}$, which implies the first part of Lemma 3.10.

To prove the second part, let $\mathbf{K}$ be the matrix with the property that

$$
\mathbf{K}_{i}= \begin{cases}\mathbf{L}_{i}^{-} & \text {if } i \in W \\ \mathbf{L}_{i}^{+} & \text {otherwise. }\end{cases}
$$

The first part of Lemma 3.10 implies that $\operatorname{det} \mathbf{K}=0$ (here we need the fact that $U$ and $W$ are disjoint). Expand $\operatorname{det} \mathbf{K}$ :

$$
\begin{aligned}
0 & =\operatorname{det} \mathbf{K}=\sum_{J \subseteq[n]}(-1)^{|W \backslash J|} \operatorname{det} \mathbf{L}_{J} \\
& =(-1)^{|W|} \sum_{J \subseteq[n]}(-1)^{|W \cap J|} \operatorname{det} \mathbf{L}_{J} .
\end{aligned}
$$

By Theorem 3.2, $\operatorname{det} \mathbf{L}_{J}=1$ if $\pi \tau_{J}$ is an Euler trail and 0 otherwise. Note that

$$
\operatorname{sgn}\left(\left(\left(\pi \tau_{J}\right)^{-1} \pi\right)^{h^{-1}(W)}\right)=\operatorname{sgn}\left(\prod_{j \in W \cap J}(+j,-j)\right)=(-1)^{|W \cap J|} .
$$


Hence (6) implies the last part of Lemma 3.10.

Lemma 3.10 is in fact a special case of Theorem 4.1, a result that will be stated and proved in the next section. It will appear that the last statement in Lemma 3.10 remains true with the weaker assumption that $U \neq W$.

\section{Partitioning Euler trails into sets of the same size}

We prove some results about how to divide the set of Euler trails in a digraph into smaller sets of the same size.

\subsection{Main results}

Let $G$ be an Eulerian digraph $G$ with vertex set $V$. Suppose that there exist nonempty vertex sets $U_{1}, \ldots, U_{\varphi}, W_{1}, \ldots, W_{\varphi} \varsubsetneqq V$ such that

$$
V=\coprod_{k=1}^{\varphi} U_{k}=\coprod_{k=1}^{\varphi} W_{k}
$$

( $\coprod$ denotes disjoint union) and

$$
t^{-1}\left(U_{k}\right)=h^{-1}\left(W_{k}\right)
$$

for $k \in[\varphi]$, that is, all arcs starting in $U_{k}$ have their heads in $W_{k}$ (we do not require that $U_{k}$ and $W_{k}$ are disjoint). Another way of describing the situation is as follows. Let $\pi$ be any $G$-permutation, and put $A_{k}=t^{-1}\left(U_{k}\right)=h^{-1}\left(W_{k}\right)$. Then for every $\tau \in T_{G}$ and $k \in[\varphi]$,

$$
\pi \tau \pi^{-1}\left(A_{k}\right)=\tau\left(A_{k}\right)=A_{k} .
$$

THEOREM 4.1. Let $G$ be an Eulerian digraph with vertex set $V$ and arc set $A$ such that $\left|h^{-1}(v)\right| \neq 1$ for each vertex $v \in V$. Let $E_{G}$ denote the set of Euler trails in $G$. Suppose that $A=\coprod_{k=1}^{\varphi} A_{k}$ is a partition of A satisfying (8) with $A_{k}$ nonempty and with $\varphi>1$. Let $K$ be a nonempty proper subset of $[\varphi]$, and put $A^{-}=\bigcup_{k \in K} A_{k}$. Then, for any $G$-permutation $\pi$,

$$
\sum_{\sigma \in E_{G}} \operatorname{sgn}\left(\left(\sigma^{-1} \pi\right)^{A^{-}}\right)=0
$$

That is, there are just as many Euler trails $\sigma$ in $G$ such that the restriction $\left(\sigma^{-1} \pi\right)^{A^{-}}$to $A^{-}$is odd as there are Euler trails $\sigma$ such that $\left(\sigma^{-1} \pi\right)^{A^{-}}$is even.

Theorem 4.1 will be proved in Subsection 4.2. However, before we proceed, we will state another version of Theorem 4.1 . 
THEOREM 4.2. Let the conditions in Theorem 4.1 be satisfied for the Eulerian digraph $G$. For any set $M \subseteq S_{A}$, let $E(M)=E_{A}(M)$ denote the set of cyclic permutations in $M$. Let $T_{0}$ be the subgroup of $T_{G}$ consisting of all permutations $\tau \in S_{A}$ such that the restriction of $\tau$ to $A_{k}$ is an even permutation for every $k \in[\varphi-1]$. Then for any two $G$-permutations $\pi$ and $\sigma$,

$$
\left|E\left(\pi T_{0}\right)\right|=\left|E\left(\sigma T_{0}\right)\right| .
$$

In particular,

$$
\left|E\left(\pi T_{0}\right)\right|=2^{-(\varphi-1)} \cdot\left|E_{G}\right|
$$

for every G-permutation $\pi$.

For any signs $s_{1}, \ldots, s_{\varphi-1} \in\{-1,1\}$, there is a permutation $\tau \in T_{G}$ such that for all $i$ the restriction of $\tau$ to $A_{i}$ has sign $s_{i}$. Namely, $\left|h^{-1}(v)\right| \neq 1$ for every vertex $v \in V$. In particular, $T_{0}$ divides $T_{G}$ into $2^{\varphi-1}$ cosets. Hence the second statement in Theorem 4.2 is a consequence of the first statement. In Subsection 4.3, we prove Theorem 4.2 using Theorem 4.1.

In the case of $G$ being a 2-regular digraph, Theorem 4.2 can be refined as follows. Recall that the vertices $u$ and $w$ intersect in an Euler trail $\pi$ if $\pi \circ(+u,-u)(+w,-w)$ is an Euler trail; $h^{-1}(u)=\{+u,-u\}$ and $h^{-1}(w)=$ $\{+w,-w\}$.

Theorem 4.3. Let the conditions in Theorem 4.1 be satisfied for the 2regular Eulerian digraph $G$, and let $T_{0}$ be as in Theorem 4.2. Let $u$ and $w$ be vertices such that there is an arc with tail $u$ and head $w$. Then for any two $G$-permutations $\pi$ and $\sigma$, the number of Euler trails in $\pi T_{0}$ such that $u$ and $w$ intersect is equal to the number of Euler trails in $\sigma T_{0}$ such that $u$ and $w$ intersect.

With the convention that $u$ always intersects itself, the result remains valid if $u=w$. By Theorem 4.2, Theorem 4.3 is equivalent to the following statement: For any two $G$-permutations $\pi$ and $\sigma$, the number of Euler trails in $\pi T_{0}$ such that $u$ and $w$ do not intersect is equal to the number of Euler trails in $\sigma T_{0}$ such that $u$ and $w$ do not intersect. We will prove Theorem 4.3 by verifying this statement in Subsection 4.4.

\subsection{Proof of Theorem 4.1}

We recall that Theorem 4.1 considers a partition $A=\bigsqcup_{j=1}^{\varphi} A_{j}$ satisfying (8) and a set $A^{-}=\bigcup_{k \in K} A_{k}$, where $K \varsubsetneqq[\varphi]$ is nonempty. Put $A^{+}=A \backslash A^{-}$, and let $\pi$ be a fixed $G$-permutation; we may without loss of generality assume that $\pi$ is an Euler trail. Our first goal is to show how the problem can be reduced to the case $\pi\left(A^{-}\right)=A^{+}$. 
Put $B^{+}=\pi^{-1}\left(A^{-}\right) \cap A^{+}, B^{-}=\pi^{-1}\left(A^{+}\right) \cap A^{-}$, and $B=B^{+} \cup B^{-}$. By (8), this definition of $B^{+}$and $B^{-}$does not depend on the choice of $\pi ; B^{+}$is the set of $\operatorname{arcs} b$ with the property that the arcs with tail $h(b)$ belong to $A^{-}$. This implies for every $v \in V$ that if $h^{-1}(v) \cap B \neq \phi$, then $h^{-1}(v) \subset B$. Hence $\left|h^{-1}(v) \cap B\right| \neq 1$. Note that $\pi^{-1}\left(A^{-}\right) \neq A^{-}$. Namely, since $\pi$ is a cyclic permutation, equality would imply that $A^{-}=A$. In particular, $B^{+}$and $B^{-}$are nonempty.

Consider a $G$-permutation $\sigma$, and recall the construction of $\sigma^{B}$ from (1). Put $T_{B}=T_{G} \cap S_{B}$. Since $\rho T_{B}=\sigma T_{B}$ if $\rho \in \sigma T_{B}$,

$$
E_{G}=E_{A}\left(\pi T_{G}\right)=\coprod_{i=1}^{m} E_{A}\left(\sigma_{i} T_{B}\right)
$$

for some $\sigma_{1}, \ldots, \sigma_{m} \in E_{G} ; E_{A}(M)$ is the set of cyclic permutations in $M \subseteq$ $S_{A}$. Thus it suffices to show that

$$
\sum_{\rho \in E_{A}\left(\sigma_{i} T_{B}\right)} \operatorname{sgn}\left(\left(\rho^{-1} \pi\right)^{A^{-}}\right)=0
$$

for every $i \in[m]$. Let $G_{i}$ be the digraph induced by $\sigma_{i}^{B} T_{B} \subset S_{B}$ (see Section 2). This means that $T_{G_{i}}=T_{B}$ (restricted to $S_{B}$ ) and that $\sigma_{i}^{B}$ is an Euler trail in $G_{i}$. An important observation is that $\rho \in \sigma_{i} T_{B}$ is an Euler trail in $G$ if and only if $\rho^{B}$ is an Euler trail in $G_{i}$. Namely, if $\rho^{B}$ happens to be an Euler trail in $G_{i}$ without $\rho$ being an Euler trail in $G$, then $\rho$ contains some cycle with arcs exclusively from $A \backslash B$. However, since $\rho^{-1} \sigma_{i}$ leaves all elements in $A \backslash B$ fixed, the very same cycle will occur in $\sigma_{i}$, which is a contradiction to the fact that $\sigma_{i}$ is an Euler trail. Thus

$$
\begin{aligned}
\sum_{\rho \in E_{A}\left(\sigma_{i} T_{B}\right)} \operatorname{sgn}\left(\left(\rho^{-1} \pi\right)^{A^{-}}\right) & =\sum_{\rho \in E_{A}\left(\sigma_{i} T_{B}\right)} \operatorname{sgn}\left(\left(\rho^{-1} \sigma_{i}\right)^{A^{-}}\right) \operatorname{sgn}\left(\left(\sigma_{i}^{-1} \pi\right)^{A^{-}}\right) \\
& =\sum_{\rho^{B} \in E_{G_{i}}} \operatorname{sgn}\left(\left(\rho^{-1} \sigma_{i}\right)^{B^{-}}\right) \operatorname{sgn}\left(\left(\sigma_{i}^{-1} \pi\right)^{A^{-}}\right) .
\end{aligned}
$$

Here, the first identity is justified by the fact that all permutations are restrictions to $A^{-}$, while the second identity follows from the fact that the restriction of $\rho^{-1} \sigma_{i}$ to $A^{-} \backslash B^{-}$is the identity permutation. The conclusion is that (9) is equivalent to

$$
\sum_{\rho^{B} \in E_{G_{i}}} \operatorname{sgn}\left(\left(\rho^{-1} \sigma_{i}\right)^{B^{-}}\right)=0 .
$$

Note that the conditions in Theorem 4.1 are satisfied if $G, A, A^{-}$, and $\pi$ are replaced by $G_{i}, B, B^{-}$, and $\sigma_{i}^{B}$, respectively. Namely, one easily convinces 
oneself that for every $\rho \in \sigma_{i} T_{B},\left(\rho^{B}\right)^{-1}\left(B^{+}\right)=B^{-}$. Moreover, as we have already mentioned, $B^{+}$and $B^{-}$are nonempty and $\left|h^{-1}(v) \cap B\right| \neq 1$ for all $v \in V$. Thus we may assume that $A=B$; that is, $\pi\left(A^{-}\right)=A^{+}$.

Our aim is to reduce the problem to the situation in Lemma 3.10. Let $a \mapsto(u, w)$ mean that the tail of $a$ is $u$ and the head of $a$ is $w$. Choose an arbitrary arc $d_{0} \in A^{-}$; we have $d_{0} \mapsto\left(u_{1}, w_{0}\right)$ for some $u_{1} \in h^{-1}\left(A^{+}\right)$and $w_{0} \in h^{-1}\left(A^{-}\right)$. The set $t^{-1}\left(u_{1}\right)$ contains at least one arc $d_{-1}$ different from $d_{0}$; assume that $d_{-1} \mapsto\left(u_{1}, w_{1}\right)$. The set $h^{-1}\left(w_{1}\right)$ contains at least one member $d_{1}$ different from $d_{-1}$; assume that $d_{1} \mapsto\left(u_{2}, w_{1}\right)$. Find an $\operatorname{arc} d_{-2} \in t^{-1}\left(u_{2}\right)$ in the same manner as we found $d_{-1}$ (that is, $d_{-2} \neq d_{1}$ and $d_{-2} \mapsto\left(u_{2}, w_{2}\right)$ for some $w_{2}$ ). Continue in this zig-zag manner until for the first time $u_{k+1}=u_{j+1}$ or $w_{k}=w_{j}$ for some $j, k, k>j$. There is no loss of generality assuming that $j=0$. In Figure 2, the case $w_{3}=w_{0}$ is illustrated. The reader may note the similarities between this figure and Figure 1 in Subsection 3.3.

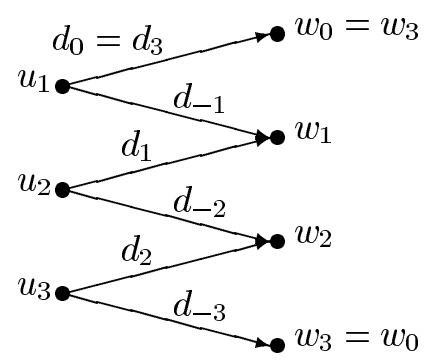

Figure 2. The case $w_{3}=w_{0}$

Put $U=\left\{u_{1}, \ldots, u_{k}\right\}, W=\left\{w_{1}, \ldots, w_{k}\right\}$, and

$$
D^{-}=\left\{d_{1}, \ldots, d_{k}, d_{-1}, \ldots, d_{-k}\right\} \subseteq A^{-} .
$$

For the Euler trail $\sigma$, put

$$
D_{\sigma}^{+}=\rho^{-1}\left(D^{-}\right) \subseteq A^{+}
$$

and $D_{\sigma}=D_{\sigma}^{+} \cup D^{-}$. Suppose that $\rho \in \sigma T_{D_{\sigma}}$, where $T_{D_{\sigma}}=T_{G} \cap S_{D_{\sigma}}$. Then $\rho(a)=\sigma(a)$ if $a \in A^{+} \backslash D_{\sigma}^{+}$, which implies that $\rho\left(D_{\sigma}^{+}\right)=\sigma\left(D_{\sigma}^{+}\right)=D^{-}$ since $\rho\left(A^{+}\right)=\sigma\left(A^{+}\right)$. Hence $D_{\rho}=D_{\sigma}$ and $\rho T_{D_{\rho}}=\sigma T_{D_{\sigma}}$. In particular, there are Euler trails $\sigma_{1}, \ldots, \sigma_{q}$ such that $E_{G}=\coprod_{j=1}^{q} E_{A}\left(\sigma_{j} T_{D_{\sigma_{j}}}\right)$. Therefore it suffices to prove

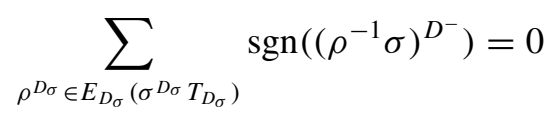


for any $\sigma \in E_{G}$ by computations similar to those implying (10). However, since $\sigma^{D_{\sigma}} T_{D_{\sigma}}$ induces a 2-regular Eulerian digraph satisfying the conditions in Lemma 3.10, (11) follows immediately.

\subsection{Proof of Theorem 4.2}

To prove Theorem 4.2, we need some names on the $2^{\varphi-1}$ different cosets in $T_{G}$ given by $T_{0}$. First we fix a $G$-permutation $\pi$. For a vector $\mathbf{y}=\left(y_{1}, \ldots, y_{\varphi-1}\right)$ of elements from $\{0,1\}$, let $E(\mathbf{y})$ be the set of Euler trails $\sigma$ such that the sign of the permutation $\left(\sigma^{-1} \pi\right)^{A_{k}}$ is equal to $(-1)^{y_{k}}$ for each $k \in[\varphi-1]$. We want to prove that $|E(\mathbf{y})|$ is the same for all vectors $\mathbf{y}$. Therefore, let $\mathbf{y}=\left(y_{1}, \ldots, y_{\varphi-1}\right)$ and $\mathbf{y}^{\prime}=\left(y_{1}^{\prime}, \ldots, y_{\varphi-1}^{\prime}\right)$ be different vectors in $\{0,1\}^{\varphi-1}$. Consider the sum

$$
\sum_{\mathbf{x} \cdot\left(\mathbf{y}^{\prime}-\mathbf{y}\right) \equiv 1}\left(\sum_{(\mathbf{z}-\mathbf{y}) \cdot \mathbf{x} \equiv 0}|E(\mathbf{z})|-\sum_{(\mathbf{z}-\mathbf{y}) \cdot \mathbf{x} \equiv 1}|E(\mathbf{z})|\right),
$$

where the outer and inner summations range over all $\{0,1\}^{\varphi-1}$-vectors $\mathbf{x}$ and $\mathbf{z}$, respectively, satisfying the indicated relations; the dot products are computed modulo 2. Let $\mathbf{x} \neq 0$ be a fixed vector; put $A^{-}=\bigcup_{x_{i}=1} A_{i}$ and $A^{+}=A \backslash A^{+}$. Note that $A^{+}$is nonempty since $A_{\varphi} \subseteq A^{+}$. Theorem 4.1 implies that

$$
\sum_{(\mathbf{z}-\mathbf{y}) \cdot \mathbf{x} \equiv 0}|E(\mathbf{z})|-\sum_{(\mathbf{z}-\mathbf{y}) \cdot \mathbf{x} \equiv 1}|E(\mathbf{z})|=0 .
$$

Namely, let $\sigma$ be such that $\operatorname{sgn}\left(\left(\sigma^{-1} \pi\right)^{A_{i}}\right)=(-1)^{y_{i}}$ for $1 \leq i \leq \varphi-1$. The first sum counts the number of Euler trails $\rho$ such that $\left(\rho^{-1} \sigma\right)^{A^{-}}$is an even permutation, while the second sum counts the other kind of permutations. In particular, (12) vanishes. We want to prove that (12) is equal to

$$
2^{\varphi-2} \cdot\left(|E(\mathbf{y})|-\left|E\left(\mathbf{y}^{\prime}\right)\right|\right)
$$

$2^{\varphi-2}$ is the number of vectors $\mathbf{x}$ such that $\mathbf{x} \cdot\left(\mathbf{y}^{\prime}-\mathbf{y}\right) \equiv 1$. Namely, this will imply that $|E(\mathbf{y})|=\left|E\left(\mathbf{y}^{\prime}\right)\right|$, which is exactly what we want to prove.

Obviously, the coefficient in front of $|E(\mathbf{y})|$ in (12) is equal to $2^{\varphi-2}$, while the coefficient of $\left|E\left(\mathbf{y}^{\prime}\right)\right|$ is equal to $-2^{\varphi-2}$. Now, let $\mathbf{z}$ be a vector such that $\mathbf{z} \neq \mathbf{y}, \mathbf{y}^{\prime}$. The coefficient of $|E(\mathbf{z})|$ is computed as follows. Since $\mathbf{z}-\mathbf{y}$ and $\mathbf{y}^{\prime}-\mathbf{y}$ are linearly independent over $G F(2)$, there is a vector $\mathbf{x}^{\prime}$ such that $(\mathbf{z}-\mathbf{y}) \cdot \mathbf{x}^{\prime} \equiv 1$ $(\bmod 2)$ and $\left(\mathbf{y}^{\prime}-\mathbf{y}\right) \cdot \mathbf{x}^{\prime} \equiv 0(\bmod 2)$. Note that $\mathbf{x} \mapsto\left(\mathbf{x}+\mathbf{x}^{\prime}\right) \bmod 2$ is a permutation of the set consisting of vectors $\mathbf{x}$ such that $\mathbf{x} \cdot\left(\mathbf{y}^{\prime}-\mathbf{y}\right) \equiv 1(\bmod$ 
2). Thus since

$$
\begin{aligned}
-\sum_{\mathbf{x} \cdot\left(\mathbf{y}^{\prime}-\mathbf{y}\right) \equiv 1}(-1)^{(\mathbf{z}-\mathbf{y}) \cdot \mathbf{x}} & =(-1)^{(\mathbf{z}-\mathbf{y}) \cdot \mathbf{x}^{\prime}} \cdot \sum_{\mathbf{x} \cdot\left(\mathbf{y}^{\prime}-\mathbf{y}\right) \equiv 1}(-1)^{(\mathbf{z}-\mathbf{y}) \cdot \mathbf{x}} \\
& =\sum_{\mathbf{x} \cdot\left(\mathbf{y}^{\prime}-\mathbf{y}\right) \equiv 1}(-1)^{(\mathbf{z}-\mathbf{y}) \cdot\left(\mathbf{x}+\mathbf{x}^{\prime}\right)} \\
& =\sum_{\left(\mathbf{x}+\mathbf{x}^{\prime}\right) \cdot\left(\mathbf{y}^{\prime}-\mathbf{y}\right) \equiv 1}(-1)^{(\mathbf{z}-\mathbf{y}) \cdot\left(\mathbf{x}+\mathbf{x}^{\prime}\right)} \\
& =\sum_{\mathbf{x} \cdot\left(\mathbf{y}^{\prime}-\mathbf{y}\right) \equiv 1}(-1)^{(\mathbf{z}-\mathbf{y}) \cdot \mathbf{x}}
\end{aligned}
$$

the coefficient in front of $|E(\mathbf{z})|$ is 0 . The theorem is proved.

\subsection{Proof of Theorem 4.3}

By assumption there is an arc, say $+w$, with tail $u$ and head $w$. Say that the tail of the second arc $-w$ with head $w$ is $u^{\prime}$, and let $a$ be the second arc with tail $u$; say that the head of $a$ is $w^{\prime}$. If $u=w$, then there is nothing to prove, since in this case $\pi \circ(+u,-u)(+w,-w)=\pi$ for all Euler trails $\pi$. If $u=u^{\prime}$ then there are two arcs from $u$ to $w$, and it is obvious that $u$ and $w$ intersect in all Euler trails. If instead $u=w^{\prime}$ or $u^{\prime}=w$, then there will be a loop at $u$ or $w$, which means that $u$ and $w$ never intersect in any Euler trail. Thus assume that $u, w, u^{\prime}, w^{\prime}$ are all different.

Let $H$ be the 2-regular Eulerian digraph obtained from $G$ as follows: We remove the vertex $w$ and the arcs $+w,-w$ from $G$. Moreover, the arcs with tail $w$ in $G$ will have $u$ as their tail in $H$. Finally, the $\operatorname{arc} a$, whose tail is $u$ in $G$, will have $u^{\prime}$ as its tail in $H$. The situation is illustrated in Figure 3.

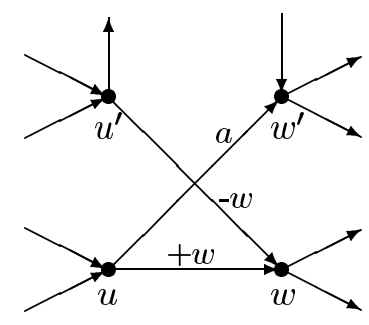

$G$
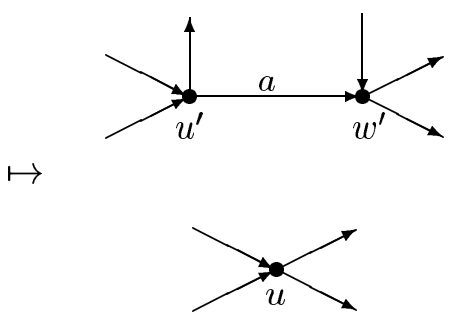

$H$

Figure 3. The construction of $H$ from $G$

Consider an Euler trail $\pi$ in $G$ where $u$ and $w$ do not intersect. This means that $\pi$ is of the form

$$
\left(a, \mathbf{s}_{1}, \alpha u,+w, \mathbf{s}_{2},-w, \mathbf{s}_{3},-\alpha u\right),
$$


where $\mathbf{s}_{i}$ are some sequences of arcs and $\alpha$ is +1 or -1 . $\pi$ can be divided into three blocks: $\left[a, \mathbf{s}_{1}, \alpha u\right],\left[+w, \mathbf{s}_{2},-w\right]$, and $\left[\mathbf{s}_{3},-\alpha u\right]$. Removing $+w$ and $-w$ and swapping block 2 and block 3 , we obtain the permutation

$$
\hat{\pi}=\left(a, \mathbf{s}_{1}, \alpha u, \mathbf{s}_{3},-\alpha u, \mathbf{s}_{2}\right),
$$

which is an Euler trail in $H$. Since the blocks can be recovered from $\hat{\pi}$ and since any Euler trail in $H$ is of the form (13), we have obtained a one-to-one correspondence between the set of Euler trails in $G$ where $u$ and $w$ do not intersect and the set of Euler trails in $H$.

We want to find sets $\hat{U}_{1}, \ldots, \hat{U}_{\varphi}$ and $\hat{W}_{1}, \ldots, \hat{W}_{\varphi}$ in $H$ satisfying (7); recall that $U_{k}=t_{G}^{-1}\left(A_{k}\right)$ and $W_{k}=h_{G}^{-1}\left(A_{k}\right)$. Put $\hat{W}_{k}=W_{k} \backslash\{w\}$. Construct the sets $\hat{U}_{k}$ by first removing $u$ and $w$ and then adding $u$ to $\hat{U}_{j}$, where $U_{j}$ is the set containing $w$. One readily verifies from this construction that (7) is satisfied for these sets in $H$. Put $\hat{A}^{k}=h^{-1}\left(\hat{W}_{k}\right)$ and $\hat{A}=A \backslash\{+w,-w\}$.

Let $\hat{T}_{0}$ be the subgroup $T_{H}$ consisting of all permutations $\tau \in S_{\hat{A}}$ such that the restriction of $\tau$ to $\hat{A}^{k}$ is an even permutation for every $k \in[\varphi-1]$. By Theorem 4.2 we have for any $H$-permutations $\hat{\pi}$ and $\hat{\sigma}$ that

$$
\left|E_{\hat{A}}\left(\hat{\pi} \hat{T}_{0}\right)\right|=\left|E_{\hat{A}}\left(\hat{\pi} \hat{T}_{0}\right)\right| .
$$

Fix an Euler trail $\pi$ in $G$ where $u$ and $w$ do not intersect; $\pi$ corresponds to the permutation $\hat{\pi}$ in the manner described above. For a vector $\mathbf{y}=\left(y_{1}, \ldots, y_{\varphi-1}\right)$ of elements from $\{0,1\}$, let $\hat{E}(\mathbf{y})$ be the set of Euler trails $\hat{\sigma}$ in $H$ such that the sign of the permutation $\left(\hat{\sigma}^{-1} \hat{\pi}\right)^{\hat{A}^{k}}$ is $(-1)^{y_{k}}$ for each $k \in[\varphi-1]$. Let $E^{*}(\mathbf{y})$ be the set of Euler trails $\sigma$ in $G$ where $u$ and $w$ do not intersect such that the sign of the permutation $\left(\sigma^{-1} \pi\right)^{A^{k}}$ is $(-1)^{y_{k}}$ for each $k \in[\varphi-1]$. Finally, let $E(\mathbf{y})$ be as in the proof of Theorem 4.2.

Suppose that $u \in W_{i}$ and $w \in W_{j} ; i$ and $j$ might be equal. Let $\mathbf{z}=$ $\left(z_{1}, \ldots, z_{\varphi-1}\right)$ be defined by $z_{k}=0$ if $k \neq i, j, z_{i}=z_{j}=1$ if $i \neq j$, and $z_{i}=z_{j}=0$ if $i=j$. To prove Theorem 4.3, it suffices to prove that

$$
\left|E^{*}(\mathbf{y})\right|+\left|E^{*}(\mathbf{y}+\mathbf{z})\right|=\left|E^{*}\left(\mathbf{y}^{\prime}\right)\right|+\left|E^{*}\left(\mathbf{y}^{\prime}+\mathbf{z}\right)\right|
$$

for every $\mathbf{y}, \mathbf{y}^{\prime} \in\{0,1\}^{\varphi-1}$, where the vector sums are computed modulo 2 . Namely, there is an obvious bijection between $E(\mathbf{y}) \backslash E^{*}(\mathbf{y})$ and $E(\mathbf{y}+\mathbf{z}) \backslash$ $E^{*}(\mathbf{y}+\mathbf{z})$ given by $\pi \mapsto \pi \circ(+u,-u)(+w,-w)$. Hence (15) and Theorem 4.2 imply Theorem 4.3.

Consider an Euler trail $\sigma$ in $G$ where $u$ and $w$ do not intersect. The sign of $\left(\sigma^{-1} \pi\right)^{A_{k}}$ is equal to the sign of $\left(\hat{\sigma}^{-1} \hat{\pi}\right)^{\hat{A}^{k}}$ if $k \neq i, j$. Namely, the procedure $\sigma \mapsto \hat{\sigma}$ only modifies the successors of $u, u^{\prime}$, and $w$, and they are all in $A_{i} \cup A_{j}$. 
This means exactly that

$$
\begin{aligned}
\left|E^{*}(\mathbf{y})\right|+\left|E^{*}(\mathbf{y}+\mathbf{z})\right|= & |\hat{E}(\mathbf{y})|+|\hat{E}(\mathbf{y}+\mathbf{z})| \\
& =\left|\hat{E}\left(\mathbf{y}^{\prime}\right)\right|+\left|\hat{E}\left(\mathbf{y}^{\prime}+\mathbf{z}\right)\right|=\left|E^{*}\left(\mathbf{y}^{\prime}\right)\right|+\left|E^{*}\left(\mathbf{y}^{\prime}+\mathbf{z}\right)\right|,
\end{aligned}
$$

where the second equality follows from (14). Theorem 4.3 is proved.

\section{2-regular arc digraphs}

We consider arc digraphs and give interpretations of Theorems 4.2 and 4.3; we will concentrate on 2-regular digraphs. The section is concluded with an application of the results to de Bruijn sequences.

\subsection{Interpretations of Theorem 4.2}

The arc digraph $K(G)$ of a digraph $G$ is defined by $V_{K(G)}=A_{G}$,

$$
A_{K(G)}=\left\{(a, b): a, b \in A_{G}, h_{G}(a)=t_{G}(b)\right\},
$$

$t_{K(G)}((a, b))=a$, and $h_{K(G)}((a, b))=b$. That is, the arcs in $G$ are the vertices in $K(G)$ and there is an arc from $a$ to $b$ in $K(G)$ if and only if the head of $a$ is equal to the tail of $b$ in $G$.

We obtain in a natural way sets $A_{1}, \ldots, A_{\varphi}$ in $K(G)$ satisfying (8), where $\varphi$ is the number of vertices in $G$. Namely, for each $k \in V_{G}$, we put

$$
A_{k}=t_{K(G)}^{-1}\left(h_{G}^{-1}(k)\right)=h_{K(G)}^{-1}\left(t_{G}^{-1}(k)\right),
$$

that is, $A_{k}$ consists of all arcs $(a, b)$ in $K(G)$ such that the head of $a$ and the tail of $b$ in $G$ is $k$. Putting $U_{k}=h_{G}^{-1}(k)$ and $W_{k}=t_{G}^{-1}(k)$, we may notice the similarity between (16) and (7). The local shape of a 2-regular arc digraph is illustrated in Figure 4.
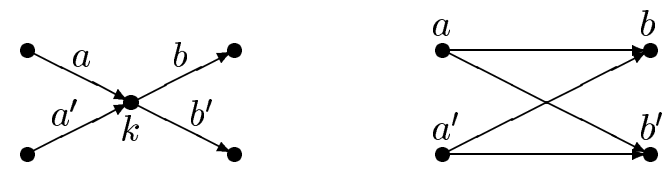

FIgURE 4. A vertex $k$ in $G$ and the corresponding $\operatorname{arc} \operatorname{set} A_{k}$ in $K(G)$

From our point of view, the most interesting result about 2-regular arc digraphs and Euler trails is the following striking correspondence between the numbers of Euler trails in a digraph and its arc digraph.

Theorem 5.1 (de Bruijn [3]; see [7]). If $G$ is a 2-regular Eulerian digraph, then

$$
\left|E_{K(G)}\right|=2^{\varphi-1}\left|E_{G}\right|,
$$


where $\varphi$ is the number of vertices in $G$.

Theorem 5.1 can be proved by using the BEST Theorem ([1]), which relates the number of Euler trails and oriented subtrees in a digraph; see also [9]. Theorems 4.2 and 5.1 imply

THEOREM 5.2. Let $G$ be a 2-regular Eulerian digraph with vertex set $[\varphi]$, and let $A_{k}$ be defined as in (16) for $k \in[\varphi]$. Let $T_{0}$ be the group of permutations $\tau \in T_{K(G)}$ such that $\operatorname{sgn}\left(\tau^{A_{k}}\right)=1$ for $k \in[\varphi-1]$. Then

$$
\left|E_{A_{K(G)}}\left(\pi T_{0}\right)\right|=\left|E_{G}\right|
$$

for any $K(G)$-permutation $\pi$.

Proof. By Theorem 4.2,

$$
\left|E_{A_{K(G)}}\left(\pi T_{0}\right)\right|=2^{\varphi-1}\left|E_{K(G)}\right|
$$

hence Theorem 5.2 is a consequence of Theorem 5.1.

The interpretation of Theorem 4.3 is somewhat more delicate.

THEOREM 5.3. Use the same notations as in Theorem 5.2, and let $a$ and $b$ be arcs in $G$ such that the head of a equals the tail of $b$. Then for any $G$ permutation $\pi$, the number of Euler trails in $E_{A_{K(G)}}\left(\pi T_{0}\right)$ such that the vertices $a$ and $b$ in $K(G)$ intersect is equal to the number of Euler trails $\sigma$ in $E_{G}$ such that $\sigma(a) \neq b$.

Proof. By Theorem 5.2, Theorem 5.3 is equivalent to the following statement: The number of Euler trails in $E_{A_{K(G)}}\left(\pi T_{0}\right)$ such that $a$ and $b$ do not intersect in $K(G)$ is equal to the number of Euler trails $\sigma$ in $E_{G}$ such that $\sigma(a)=b$.

Let $a^{\prime}$ be the arc with the same head as $a$ in $G$; hence $a^{\prime}$ is a vertex in $K(G)$ such that there is an arc from $a^{\prime}$ to $b$. Let $b^{\prime}$ be the arc with the same tail as $b$ in $G$, that is, there is an arc from $a$ to $b^{\prime}$ and from $a^{\prime}$ to $b^{\prime}$ in $K(G) . K(G)$ is illustrated in the left part of Figure 5. Construct a digraph $Q$ from $K(G)$ in the same manner as we constructed $H$ in the proof of Theorem 4.3: Remove the vertex $b$ and the arcs with head $b$. Moreover, the arcs with tail $b$ in $K(G)$ will have $a$ as their tail in $Q$. Finally, the arc with tail $a$ and head $b^{\prime}$ in $K(G)$ will have $a^{\prime}$ as its tail in $Q$ (and still $b^{\prime}$ as its head).

Following the proof of Theorem 4.3, we realize that the number of Euler trails in $K(G)$ such that $a$ and $b$ do not intersect is equal to $\left|E_{Q}\right|$. In $Q$, there are two arcs from $a^{\prime}$ to $b^{\prime}$. Construct the digraph $\hat{Q}$ from $Q$ by removing $b^{\prime}$ together with the two arcs from $a^{\prime}$ to $b^{\prime}$ and by letting $a^{\prime}$ be the new tail of the arcs with old tail $b^{\prime}$ in $Q$. We have that $\left|E_{Q}\right|=2\left|E_{\hat{Q}}\right|$. Namely, given an Euler trail 
$\left(+a^{\prime}, \mathbf{s}_{1},-a^{\prime}, \mathbf{s}_{2}\right)$ in $\hat{Q}$, we obtain two Euler trails $\left(+a^{\prime},+b^{\prime}, \mathbf{s}_{1},-a^{\prime},-b^{\prime}, \mathbf{s}_{2}\right)$ and $\left(+a^{\prime},-b^{\prime}, \mathbf{s}_{1},-a^{\prime},+b^{\prime}, \mathbf{s}_{2}\right)$ in $Q ; \mathbf{s}_{1}$ and $\mathbf{s}_{2}$ are sequences of arcs.

The graph $\hat{Q}$ can be obtained directly from $K(G)$ as follows. First remove $b$ and $b^{\prime}$ together with the arcs with head $b$ or $b^{\prime}$. Then let $a$ and $a^{\prime}$ be the new tails of the arcs with old tails $b$ and $b^{\prime}$, respectively (see Figure 5).

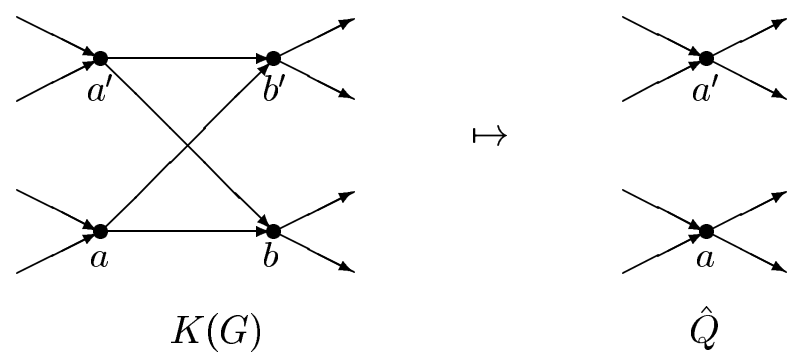

Figure 5. The construction of $\hat{Q}$ from $K(G)$

Now, construct a digraph $H$ from $G$ by removing $b, b^{\prime}$, and the head of $a$ and $a^{\prime}$; let the head of $a$ in $H$ be the head of $b$ in $G$; let the head of $a^{\prime}$ in $H$ be the head of $b^{\prime}$ in $G$. By inspection, one realizes that $K(H)=\hat{Q}$. Theorem 5.1 implies that $\left|E_{\hat{Q}}\right|=2^{\varphi-2}\left|E_{H}\right|$. Thus the number of Euler trails in $E_{K(G)}$ such that $a$ and $b$ do not intersect is equal to

$$
\left|E_{Q}\right|=2\left|E_{\hat{Q}}\right|=2^{\varphi-1}\left|E_{H}\right|,
$$

which is equal to the number of Euler trails $\sigma$ in $G$ such that $\sigma(a)=b$. Hence Theorem 5.3 follows from Theorem 4.3.

ExAmPLe 5.4. Let $G$ and $K$ be the digraphs in Figure 6; $K$ is isomorphic to the arc digraph $K(G)$ of $G$. The vertex set of $K$ is $V=$ [8] and the arc set is $A= \pm[8]$. Note that $T_{K}$ is the subgroup of $S_{A}$ generated by $(+k,-k)$, $1 \leq k \leq 8$ and that $h_{K}( \pm k)=k$.
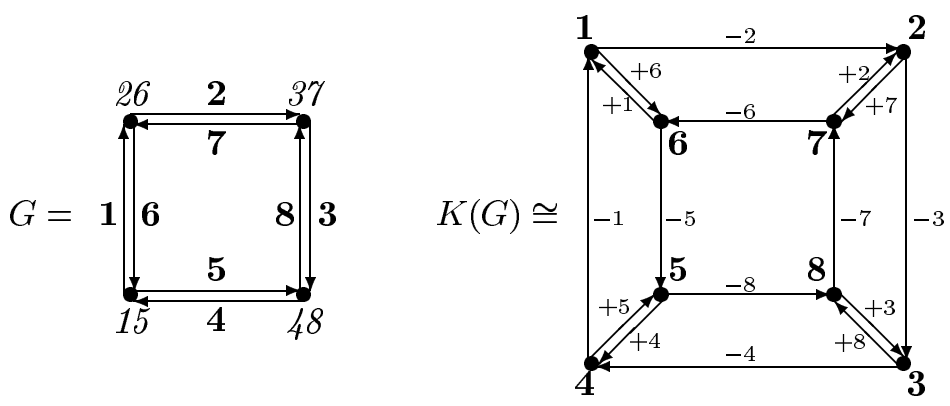

Figure 6. A graph $G$ and its arc digraph $K(G)$ 
Put $U_{1}=\{4,6\}, U_{2}=\{1,7\}, U_{3}=\{2,8\}, U_{4}=\{3,5\}$ and $W_{1}=\{1,5\}$, $W_{2}=\{2,6\}, W_{3}=\{3,7\}, W_{4}=\{4,8\}$. The names of the vertices in $G$ are chosen in correspondence to the sets $W_{1}, \ldots, W_{4} ; t_{G}^{-1}(15)=\{1,5\}$ and so on.

Consider the $K$-permutation

$$
\begin{aligned}
& \pi= \\
& \quad(-1,+6,-5,+4)(-4,+5,-8,+3)(-3,+8,-7,+2)(-2,+7,-6,+1) .
\end{aligned}
$$

For a vector $\mathbf{y}=\left(y_{1}, y_{2}, y_{3}\right) \in\{0,1\}^{3}$, let $E(\mathbf{y})$ be the set of Euler trails $\pi \tau_{J}=\pi \circ \prod_{j \in J}(+j,-j)$ such that $|J| \cap W_{k} \equiv y_{k}(\bmod 2)$. We obtain the following table, showing for each $\mathbf{y}=\left(y_{1}, y_{2}, y_{3}\right)$ all sets $J$ such that $\pi \tau_{J} \in E(\mathbf{y})$.

$$
\begin{array}{lccccc}
(0,0,0) & : & \{6,2,8\} & \{6,2,4\} & \{\mathbf{1}, \mathbf{8}, \mathbf{5}\} & \{7,3,4\} \\
(1,1,0) & : & \{1,2,8\} & \{1,2,4\} & \{6,8,5\} & \{\mathbf{2}, \mathbf{8}, \mathbf{5}\} \\
(1,0,1) & : & \{1,7,8\} & \{1,7,4\} & \{\mathbf{1}, \mathbf{3}, \mathbf{4}\} & \{7,8,5\} \\
(0,1,1) & : & \{6,7,8\} & \{6,7,4\} & \{6,3,4\} & \{\mathbf{2}, \mathbf{3}, \mathbf{4}\} \\
(1,0,0) & : & \{1,7,3\} & \{6,2,5\} & \{\mathbf{1}, \mathbf{8}, \mathbf{4}\} & \{7,3,5\} \\
(0,1,0) & : & \{6,7,3\} & \{1,2,5\} & \{6,8,4\} & \{\mathbf{2}, \mathbf{8}, \mathbf{4}\} \\
(0,0,1) & : & \{6,2,3\} & \{1,7,5\} & \{\mathbf{1}, \mathbf{3}, \mathbf{5}\} & \{7,8,4\} \\
(1,1,1) & : & \{1,2,3\} & \{6,7,5\} & \{6,3,5\} & \{\mathbf{2}, \mathbf{3}, \mathbf{5}\}
\end{array}
$$

As Theorem 5.2 states, the number of sets in each row is equal to $\left|E_{G}\right|=4$. The last column of sets corresponds to the set of Euler trails where 1 and 6 do not intersect, while the bold sets correspond to Euler trails where 1 and 2 intersect. Note that the underlying digraph $G$ contains four Euler trails, namely

$$
\begin{aligned}
& (1,6,5,8,7,2,3,4),(1,2,3,4,5,8,7,6), \\
& (1,2,7,6,5,8,3,4),(1,2,3,8,7,6,5,4) .
\end{aligned}
$$

1 is followed by 6 in one trail and by 2 in the other trails. Thus we have verified Theorem 5.3 for $K$ when $u=1$ and $w=2,6$.

\section{2. de Bruijn digraphs}

As an application of Theorem 5.2, we conclude this article with a short discussion about de Bruijn digraphs, named after the Dutch mathematician N.G. de Bruijn.

Let $m>1, b>1$. Define the digraph $G_{b, m}$ as follows. Let

$$
V=\left\{\left(c_{1}, \ldots, c_{m-1}\right): c_{1}, \ldots, c_{m-1} \in\{0, \ldots, b-1\}\right\}
$$

and

$$
A=\left\{\left(c_{1}, \ldots, c_{m}\right): c_{1}, \ldots, c_{m} \in\{0, \ldots, b-1\}\right\} .
$$


Moreover, let the tail of $\left(c_{1}, \ldots, c_{m}\right)$ be $\left(c_{1}, \ldots, c_{m-1}\right)$ and let the head be $\left(c_{2}, \ldots, c_{m}\right)$. The digraph $G_{b, m}$ is called a de Bruijn digraph. The vertices and arcs in a de Bruijn digraph can be interpreted as the $b$-ary representations of integers. For example, the vertex 011 in $G_{2,4}$ (see Figure 7) is the binary representation of $0 \cdot 4+1 \cdot 2+1 \cdot 1=3$.

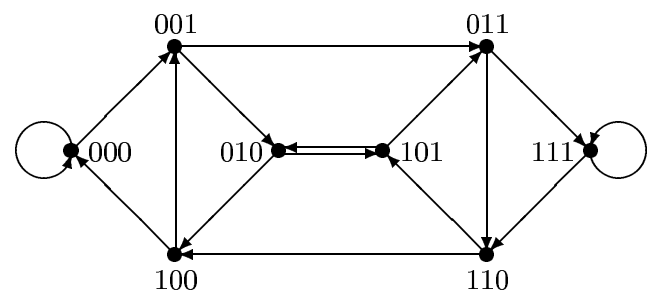

FIGURE 7. This is $G_{2,4}$; arc labels are omitted

The equivalence class

$$
\mathbf{s}=\left\langle s_{0}, \ldots, s_{k-1}\right\rangle
$$

of rotations of a sequence $\left(s_{0}, \ldots, s_{k-1}\right)$ is called a cyclic sequence. A word $\left(c_{1}, \ldots, c_{m}\right)$ is contained in $\mathbf{s}$ if there is an $i(0 \leq i<k)$ such that $s_{i+j}=c_{j}$ for $1 \leq j \leq m$ (the indices are taken modulo $k$ ). The Euler trails in $G_{b, m}$ correspond to cyclic sequences $\mathbf{S}=\left\langle s_{0}, \ldots, s_{b^{m}-1}\right\rangle$ with the property that each $b$-ary word of length $m$ is contained exactly once in $\mathbf{s}$. Such cyclic sequences are called de Bruijn sequences. For example, there are two binary de Bruijn sequences of length $2^{3}$, namely $\langle 00010111\rangle$ and $\langle 00011101\rangle$. The Euler trail corresponding to the cyclic sequence $\left\langle s_{0}, \ldots, s_{b^{m}-1}\right\rangle$ is the Euler trail $\pi \in$ $E_{G_{b, m}}$ with the property that

$$
\pi\left(s_{k}, s_{k+1}, \ldots, s_{k+m-1}\right)=\left(s_{k+1}, s_{k+2}, \ldots, s_{k+m}\right)
$$

for all $k$; the indices are computed modulo $b^{m}$.

In the following, we will only deal with the 2-regular case; therefore put $G_{m}=G_{2, m}$. For any binary sequence $\mathbf{p}=\left(p_{1}, \ldots, p_{k}\right)(k \geq 1)$ and binary numbers $x_{1}, \ldots, x_{a}, y_{1}, \ldots, y_{b}(a, b \geq 0)$, put

$$
\left(x_{1}, \ldots, x_{a}, \mathbf{p}, y_{1}, \ldots, y_{b}\right)=\left(x_{1}, \ldots, x_{a}, p_{1}, \ldots, p_{k}, y_{1}, \ldots, y_{b}\right) .
$$

One realizes that the sets

$$
U_{\mathbf{p}}=\{(0, \mathbf{p}),(1, \mathbf{p})\}
$$

and

$$
W_{\mathbf{p}}=\{(\mathbf{p}, 0),(\mathbf{p}, 1)\}
$$


satisfy (7). Moreover, it is not difficult to check that $G_{m}$ is isomorphic to $K\left(G_{m-1}\right)$, the arc digraph of $G_{m-1}$.

Let $m>2$. An $m$-de Bruijn sequence is a binary de Bruijn sequence of length $2^{m}$. Theorem 5.1 and induction imply that the number $\left|E_{G_{m}}\right|$ of $m$-de Bruijn sequences is equal to $2^{2^{m-1}-m}$ (see [7]).

THeOREM 5.5. Let

$$
\left\{y_{\mathbf{p}}: \mathbf{p}=\left(p_{1}, \ldots, p_{m-2}\right) \in\{0,1\}^{m-2}\right\}
$$

be a set of binary numbers. Then the number of $m$-de Bruijn sequences containing exactly one of the sequences $(0, \mathbf{p}, 0,0)$ and $\left(0, \mathbf{p}, 1, y_{\mathbf{p}}\right)$ for each $\mathbf{p} \in\{0,1\}^{m-2} \backslash\{(1,1, \ldots, 1)\}$ is equal to the number $2^{2^{m-2}-(m-1)}$ of $(m-1)$ de Bruijn sequences.

Proof. Let $\pi$ be the $G_{m}$-permutation given by $\pi(0, \mathbf{p}, 0)=(\mathbf{p}, 0,1)$ and $\pi(0, \mathbf{p}, 1)=\left(\mathbf{p}, 1, y_{\mathbf{p}}\right)$ for all $\mathbf{p} \in\{0,1\}^{m-2}$. Let $T_{0}$ be the group of permutations $\tau \in T_{G_{m}}$ such that the first digit in $\tau(0, \mathbf{p}, 0)$ is equal to the first digit in $\tau(0, \mathbf{p}, 1)$ for all $\mathbf{p} \in\{0,1\}^{m-2} \backslash\{(1,1, \ldots, 1)\}$, that is, $T_{0}$ is defined as in Theorem 5.2. Then $\pi T_{0}$ contains all Euler trails corresponding to $m$-de Bruijn sequences with the property in Theorem 5.5. Theorem 5.2 implies that

$$
\left|E_{A_{G_{m}}}\left(\pi T_{0}\right)\right|=\left|E_{G_{m-1}}\right|,
$$

which is equal to the number of $(m-1)$-de Bruijn sequences.

\section{REFERENCES}

1. van Aardenne-Ehrenfest, T. and de Bruijn, N. G., Circuits and trees in oriented linear graphs, Simon Stevin 28 (1951), 203-217.

2. Beck, I., Cycle decomposition by transpositions, J. Combin. Theory Ser. A 23 (1977), 198207.

3. de Bruijn, N. G., A combinatorial problem, Indag. Math. 8 (1946), 461-467.

4. Cohn, M. and Lempel, A., Cyclic decomposition by disjoint transpositions, J. Combin. Theory Ser. A 13 (1972), 83-89.

5. Dixon, J. D. and Mortimer, B., Permutation Groups, Springer Verlag, 1996.

6. Faddeev, D. K. and Faddeeva, V. N., Computational Methods of Linear Algebra, W. H. Freeman and Company, 1963.

7. Hall, Marshall Jr, Combinatorial Theory, Blaisdell Publishing Company, 1967.

8. Jonsson, J., Euler Trails and Trees in Directed Graphs, Technical Report, Department of Mathematics, Stockholm University (1999).

9. Knuth, D. E., Oriented subtrees of an arc digraph, J. Combin. Theory, Ser. A 3 (1967), 309-314.

10. Lauri, J., On a formula for the number of Euler trails for a class of digraphs, Discrete Math. 163 (1997), 307-312.

11. Macris, N. and Pulé, J. V., Density of states of random Schrödinger operators with a uniform magnetic field, Lett. Math. Phys. 24 (1992), 307-321. 
12. Macris, N., and Pulé, J. V., An alternative formula for the number of Euler trails for a class of digraphs, Discrete Math. 154 (1996), 301-305.

DEPARTMENT OF MATHEMATICS

UNIVERSITY OF STOCKHOLM

S-106 91 STOCKHOLM

SWEDEN

E-mail: jacob@matematik.su.se 